\title{
Vessel Extraction for AS-OCT Angiography
}

\author{
Huazhu $\mathrm{Fu}^{1}$, Yanwu Xu${ }^{1}$, Damon Wing Kee Wong ${ }^{1}$, Marcus Ang ${ }^{2}$, \\ Suchandrima Das ${ }^{2}$, and Jiang $\mathrm{Liu}^{3}$ \\ ${ }^{1}$ Institute for Infocomm Research, A*STAR, Singapore. \\ ${ }^{2}$ Singapore Eye Research Institute and Singapore National Eye Centre, Singapore. \\ ${ }^{3}$ Cixi Institute of Biomedical Engineering, Ningbo Institute of Materials Technology \\ and Engineering, Chinese Academy of Sciences, China.
}

\begin{abstract}
In this work, we propose a filter-based vessel segmentation method for Anterior Segment Optical Coherence Tomography Angiography image. In our method, the bandpass filter is utilized to suppress the horizontal noise lines caused by eye movement, while the curvedsupport Gaussian filter is utilized to enhance the vessel and generate the probability map.
\end{abstract}

\section{Introduction}

Optical Coherence Tomography Angiography (OCTA) is a new non-invasive imaging technique that employs motion contrast imaging to high-resolution volumetric blood flow information generating angiographic images in a matter of seconds [4]. OCTA has been used to successfully delineate vessels within the retina, choroid and the vasculature associated with the optic nerve. Recently, the clinical studies have demonstrated the OCTA can be adapted for the assessment of anterior segment vasculature $[2,1]$, where the vessel measurements in a small region of interest (ROI) are calculated to determinate the corneal vascularisation. Thus, the vessel segmentation is a key point for these clinical applications. However, the automatic vessel segmentation from the AS-OCT angiography is a challenge problem. For example, the eye movement during the OCT-A scanning produces motion artifacts in the form of numerous horizontal lines, as shown in Fig. 1 (A). The segmentation methods proposed here can easily remove these motion artifacts and extract the vessels for further measurements.

\section{Method}

To address above problem, we propose a filter-based vessel segmentation method for AS-OCT Angiography image. Fig. 1 illustrates the framework of our method. Given the AS-OCT Angiography image, we first do pro-processing by using the bandpass filter to suppress the horizontal noise lines caused by eye movement. We choose the Gaussian bandpass filter in our method, which is formulated as $G(u, v)=\exp -\left[\frac{D^{2}-D_{0}^{2}}{D W}\right]^{2}$, where $D$ is the distance from the center of the frequency rectangle, $D_{0}$ and $W$ are the radial center and wide of the band of

X. Chen, M. K. Garvin, J. Liu, E. Trucco, Y. Xu (Eds.): OMIA 2016, Held in Conjunction with MICCAI 2016, Athens, Greece, Iowa Research Online, pp. 158-159, 2016. Available from: http://ir.uiowa.edu/omia/2016_Proceedings/2016/ 

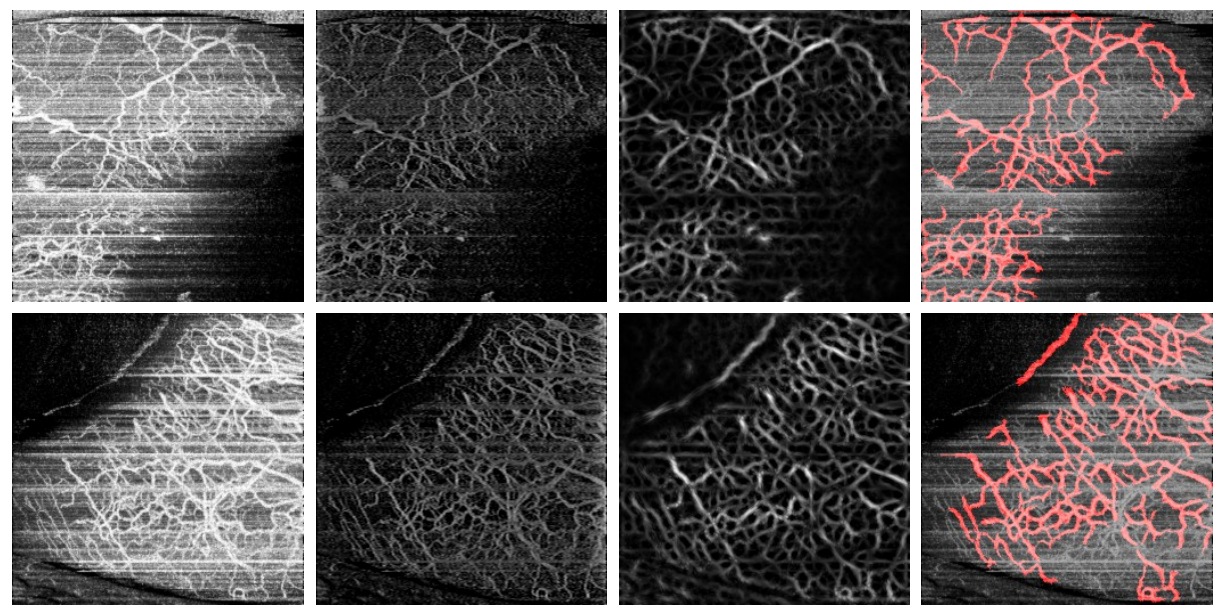

\section{(A) Input}

(B) Pro-process

(C) Vessel enhancement

(D) Segmentation

Fig. 1. From left to right: the input AS-OCT-Angiography image, the pro-processed image, the vessel probability map, the final segmentation result.

interest, respectively. An example after the bandpass filtering is shown in Fig. 1 (B). After that, the Scale and Curvature Invariant Ridge Detector (SCIRD) [3] is employed to enhance the vessel map. SCIRD is simultaneously rotation, scale and curvature invariant, and removes the assumption of locally straight tubular structures based on the curved-support Gaussian model [5]. With the SCIRD, the vessel probability map is obtained as shown in Fig. 1 (C). Finally, a thresholding is set to segment the binary vessel mask. Some visual results are shown in Fig. 1 (D), where the vessel (highlighted by red) are extracted accurately.

\section{References}

1. Ang, M., Cai, Y., MacPhee, B., Sim, D., Keane, P., Sng, C., Egan, C., Tufail, A., Larkin, D., Wilkins, M.: Optical coherence tomography angiography and indocyanine green angiography for corneal vascularisation. British Journal of Ophthalmology (2016)

2. Ang, M., Sim, D., Keane, P., Sng, C., Egan, C., Tufail, A., Wilkins, M.: Optical Coherence Tomography Angiography for Anterior Segment Vasculature Imaging. Ophthalmology 122(9), 1740-1747 (2015)

3. Annunziata, R., Kheirkhah, A., Hamrah, P., Trucco, E.: Scale and Curvature Invariant Ridge Detector for Tortuous and Fragmented Structures. In: MICCAI. pp. 588-595 (2015)

4. de Carlo, T., Romano, A., Waheed, N., Duker, J.: A review of optical coherence tomography angiography (OCTA). International Journal of Retina and Vitreous pp. $1-15(2015)$

5. Lin, J.K., Dayan, P.: Curved gaussian models with application to the modeling of foreign exchange rates. Computational Finance (1999) 